\title{
Diminishing Disparities: Starting in Our Own Backyards
}

\author{
Joan J. Ryoo, MD, Sushma Jain, MBBS, MPH, and Clifford Y. Ko, MD, MS, MSHS, FACS
}

Department of Surgery, UCLA, 10833 LeConte Ave. 72-215 CHS, Los Angeles, CA 90095, USA

Disparities in health - those "differences in the incidence, prevalence, mortality, and burden of diseases and other adverse health conditions that exist among specific population groups", as defined by the National Institutes of Health-are becoming increasingly well described in the surgical literature. The awakening to such inequities has been long in coming, if not long overdue; research endeavors pioneered in the realms of public health, internal medicine, public policy, and the social sciences have provided a substantial foundation upon which disciplines such as surgery have had the privilege to build.

In this issue of the Annals of Surgical Oncology, Greenstein et al. laudably assume this task with regard to esophageal cancer, an area in which examinations focusing on disparities are growing, but still wanting. Over several decades, differential incidence and survival have been observed according to ethnicity, with black people, in particular, suffering a strikingly higher risk not only of disease, but also of worse outcomes as compared to white people. ${ }^{1-2}$

On initial examination of the data on patients with early esophageal cancer as identified through the Surveillance, Epidemiology, and End Results (SEER) program of 1988-2003, the investigators found that five-year, disease-specific survival was significantly worse for black as opposed to white people, with histological type and $\mathrm{T}$ status being factors associated with worse survival. ${ }^{3}$ However, in subsequent analyses that controlled for histology, stage, and treatment

Received September 24, 2007; accepted September 25, 2007; published online: December 19, 2007.

Address correspondence and reprint requests to: Clifford Y. Ko, MD, MS, MSHS, FACS; E-mail: cko@mednet.ucla.edu

Published by Springer Science+Business Media, LLC @ 2007 The Society of Surgical Oncology, Inc. received (surgery versus radiation therapy), ethnic origin was no longer associated with increased disease-specific mortality $(p=0.19)$, suggesting that poorer outcomes may be associated with treatment choice. Of note, black people were significantly less likely than white people to have surgery ( $44 \%$ versus $66 \%, p<0.001)$.

Importantly, this study identifies factors that may explain differences in the crude mortality from esophageal cancer found when comparing different ethnic groups - namely, histology, stage at presentation, and therapeutic modality. The latter two predictors of inequity may be seen as potentially mutable, in that alterations or enhancements in processes of care, for instance, might help diminish disparate outcomes. Furthermore, this study underscores the possibility that disparities in esophageal cancer may result from issues associated with our health care system, including those specific to surgery.

In essence, what these results beseech is the next step in understanding the disparities experienced by black people with regard to esophageal cancer: moving from hypothesis to the testing of proposed mechanisms and identification of modifiable risk factors, such that interventions can be instituted to effect change. This requires simultaneous efforts to elucidate, on a microbiological level, the processes of carcinogenesis and, on a macrobiological level, the mechanisms underlying disparity - particularly those mutable predictors of disease and mediators of outcomes. From systems factors creating barriers to care or navigation, to provider factors, such as cultural competency and provision of quality care, to individual factors including impaired access and attitudes or beliefs, the possible determinants of inequitable 
outcomes are many and their relationships are undoubtedly complex, but -if clarified - may offer much-needed opportunities for intervention.

The challenge is, indeed, formidable, but some headway that may point to potential avenues for promoting change has already been made. In a review of the SEER registry that was linked to Medicare databases, Steyerberg et al. ${ }^{4}$ identified treatment as a potentially modifiable factor affecting outcomes for elderly individuals with locoregional esophageal cancer, demonstrating that not only were black people less likely to have a surgical evaluation but, once seen in consultation by a surgeon, were less likely to undergo surgery. When survival was adjusted for treatment received, the difference seen after stratification by ethnic origin was abrogated. Access to health care, then, may not be the sole issue, but rather (as in this case), what is seemingly disparate is decision-making once access is achieved.

Recommendations for intervention rely on identifying mutable predictors of disparity; thus, future examinations arguably should focus more on identifying factors that may be modified to prevent disparities in the first place. In the case of patientprovider discussion regarding an operation, a number of potentially mutable issues arise that may be addressed, including whether consultations are offered appropriately, whether patients are able to meet consultants, whether surgery is offered appropriately, whether providers are able to communicate effectively with patients, etc. Moreover, follow-up investigations that examine whether disparities are actually lessened by such interventions should be performed. The current study by Greenstein et al. ${ }^{3}$ does well in revealing predictors of disparity with respect to esophageal cancer, however, the next, difficult step is to figure out how to detect cancer at an earlier stage and how to intervene for the improvement of outcomes. These aims, whether researchoriented or not, are relevant not only to esophageal carcinoma, but to cancer more generally.

Examples of efforts in the field provide valuable approaches applicable to surgical oncology, including the implementation of patient navigators to improve negotiation of the health care system and follow-up processes that are extremely germane to oncologic diseases, which often involve multiple providers and require tremendous coordination by patient and systems for continuity of care. ${ }^{5}$ Education at both institutional and local levels may also help improve outcomes. One study examining knowledge of prostate cancer pre- and post-seminar revealed an increased awareness of risk among black participants, as well as willingness of over half the sample population to engage in screening following the intervention. ${ }^{6}$

Of great promise for the aim of decreasing disparities and improving outcomes is community-based participatory research (CBPR), which engages providers and populations in identifying community health priorities and taking into account not only needs at the local level, but also the tools and means with which to address those needs. Given that many health disparities occur in the community, the technique of CBPR attempts to move beyond traditional outreach alone to more balanced, partnered approaches. These approaches strive to develop mutually beneficial relationships between communities experiencing health inequities and researchers who study those disparities, with emphasis on the bidirectional nature of the relationship and alternative modes of inquiry that aspire to achieve social change in a sustainable fashion. CBPR, in ideal terms, may be envisioned as providing an opportunity for academia and communities to both improve health care and diminish disparities simultaneously.

A number of organizations have sponsored the promotion of just such relationships for communitypartnered research. In south Los Angeles, the Healthy African American Families organization has been able to conduct a number of community interventions in partnership with nearby academic institutions, including initiatives in preventive care, ${ }^{7,8}$ providing methods pertinent to surgical oncology, with regard to screening and surveillance. In the realm of oncology, the Moffitt Cancer Center has established a relationship with a community and employed a systematic approach to improving cancer awareness and screening with a CBPR program that addresses aspects of health care including culture, literacy, education, assessment, and networking (CLEAN). ${ }^{9}$ These models provide great hope and inspiration for redressing health care inequities in practice, today and tomorrow, on the ground.

In summary, disparities in health care abound. Ultimately, studies in our discipline should seek to shift future investigative efforts less toward the description of known inequities and more toward action to diminish such disparities and improve patient care.$^{10}$ Working in our own backyards may be a good place to start.

\section{REFERENCES}

1. Pickens A, Orringer MB. Geographical distribution and racial disparity in esophageal cancer. Ann Thorac Surg 2003; 76:S1367-9. 
2. Baquet CR, Commiskey P, Mack K, Meltzer S, Mishra SI. Esophageal cancer epidemiology in blacks and whites: racial and gender disparities in incidence, mortality, survival rates and histology. J Natl Med Assoc 2005; 97(11):1471-8.

3. Greenstein AJ, Litle VR, Swanson SJ, Divino CM, Packer S, McGinn TG, Wisnivesky JP. Racial disparities in esophageal cancer treatment and outcomes. Ann Surg Oncol. In press. doi:10.1245/s10434-007-9664-5.

4. Steyerberg EW, Earle CC, Neville BA, Weeks JC. Racial differences in surgical evaluation, treatment, and outcome of locoregional esophageal cancer: A population-based analysis of elderly patients. J Clin Oncol 2005; 13(3):510-7.

5. Ell K, et al. Patient navigation and case management following an abnormal mammogram: A randomized clinical trial. Prev Med 2007; 44(1):26-33.
6. Wilkinson $\mathrm{S}$, et al. Educating African-American men about prostate cancer: impact on awareness and knowledge. Urology 2003; 61(2):308-13.

7. Sarkisian CA, et al. Using focus groups of older African Americans and Latinos with diabetes to modify a self-care empowerment intervention. Ethn Dis 2005; 15(2):283-91.

8. Bluthenthal RN, et al. Witness for wellness: preliminary findings from a community-academic participatory research mental health initiative. Ethn Dis 2006; 16:S18-34.

9. Meade CD, et al. Impacting health disparities through community outreach: Utilizing the CLEAN look (culture, literacy, education, assessment, and networking). Cancer Control 2007; 14(1):70-7.

10. Lurie N. Health disparities - less talk, more action. $N$ Engl $J$ Med 2005; 353(7):727-9. 